\title{
A recursive finite element method for computing tyre vibrations
}

\author{
Denis Duhamel - Silvano Erlicher - Hong Hai Nguyen \\ Université Paris-Est, Laboratoire Navier \\ Ecole des Ponts ParisTech \\ 6 et 8 Avenue Blaise Pascal \\ Cité Descartes, Champs sur Marne \\ F-77455 Marne-la-Vallée, cedex 2 \\ denis.duhamel@enpc.fr
}

ABSTRACT. A numerical method is described for computing tyre vibrations over a large frequency range. It is based on a recursive finite element method for building the dynamic stiffness matrix of a complete tyre from the knowledge of a finite element model of a small part of the structure. The present method is compared to full three-dimensional finite element solutions showing a perfect agreement for low frequencies. However, this method allows computations for medium and high frequencies which are needed for the analysis of noise generated by a tyre. The influence of various parameters on the frequency response functions like the positions where the responses are computed, the mechanical parameters of the tyre or the internal air-pressure are described.

RÉSUMÉ. Une méthode numérique permettant le calcul des vibrations d'un pneumatique sur une large gamme de fréquences est proposée. Elle est basée sur une approche récursive pour calculer la matrice de rigidité dynamique d'un pneumatique complet à partir de la connaissance d'un modèle éléments finis d'une petite partie de la structure. La méthode proposée est comparée à un calcul tridimensionnel et montre un parfait accord pour les basses fréquences. Cette méthode permet cependant des calculs dans les moyennes et hautes fréquences qui sont nécessaires pour analyser le bruit engendré par le roulement d'un pneumatique. L'influence de paramètres variés sur les fonctions de réponse en fréquences, comme les positions des points de calcul, les paramètres mécaniques du pneumatique ou la pression de gonflement est décrite.

KEYWORDS: vibration, tyre, periodic structure, finite element, recursive.

MOTS-CLÉS : vibration, pneumatique, structure périodique, éléments finis, récursivité.

DOI:10.3166/EJCM.20.9-27 @ 2011 Lavoisier, Paris

EJCM - 20/2011. Dynamics of materials, structures and systems, pages 9 to 27 


\section{Introduction}

The modelling of tyre noise radiation requires the computation of tyre vibrations for a wide frequency range, including medium and high frequencies. This problem is difficult to solve with classical numerical methods like the sum of modes computed by the finite element method. With the present computer resources, this can be done only for low frequencies and leads to very heavy computations for higher frequencies both for the number of modes to consider and the number of degrees of freedom required to compute a single mode. Nevertheless, various methods have been proposed in the past to try to compute tyre vibrations.

The first class of methods is based on analytical models. The first model considered in the past was the circular ring model (Heckl, 1986; Huang et al., 1987; Kropp, 1989; Campanac et al., 2000) in which the tyre is described by a circular beam under tension. To be more realistic for high frequencies, more complex analytical models, mainly based on orthotropic plates, were proposed. The time domain Green's function for the orthotropic plate was computed by Hamet (Hamet, 2001). In (Larsson et al., 2002b) a high frequency model based on the coupling of two elastic layers is presented. Muggleton et al. (Muggleton et al., 2003) developed a semi-analytical model made of orthotropic plates in plane stress submitted to an internal air pressure. Two plates are connected and represent respectively the tread and the side of a tyre. The internal pressure is considered, but the curvature of the side geometry and the non uniformity of the thickness are neglected. More generally in (Pinnington et $a l .$, 2002), a four parameters model is described leading to a fourth order wave equation. In (Pinnington, 2002) a method is proposed to estimate side rigidities at various frequencies and in (Pinnington, 2006a; Pinnington, 2006b) the model was extended to include the following parameters: curvature, shear rigidity, rotary inertia, tension, rotating velocity and internal air pressure. The curvature leads to a coupling between the radial and tangential movements. A shell model was also developed by (Kim et al., 2004) to study the influence of the rotation and an analytical shell model coupled with an acoustic cavity was developed by (Molisani et al., 2003) while another shell model was developed by (Kozhevnikov, 2006). All these analytical models provide interesting insights into tyre vibrations, however, they do not allow the consideration of the detailed geometrical and material properties of a real tyre.

The other approaches were mainly numerical or a coupling of numerical and analytical models. So, Larsson et al (Larsson et al., 2002a) developed a coupled numerical and analytical model of a tyre including the bloc pattern. Blocs with simple shapes, like cubic shapes, are considered and the other parts of the tyre are modelled as an orthotropic plate. The radial mobility is improved by the inclusion of blocs in the model while the improvement is less for the tangential mobility. The full three-dimensional model of a tyre was considered by (Brinkmeier et al., 2008) but the frequency range of analysis was limited by the huge requirement in computational power needed by this type of model. A similar approach was used by (Lopez et al., 2007) but with an original method taking into account the influence of the rotation of the tyre. 
It can be useful to see tyres as circular waveguides. This leads to another interesting numerical method which is the spectral finite element method (SFE). It is based on an analytical wave decomposition along the waveguide axis and is mainly described by (Finnveden, 1997a; Finnveden, 1997b) for uniform waveguides with a complex section. In this method, the discrete equation is developed into a series of different powers of the wavelength with matrices obtained by variational methods which are different from the usual matrices of the finite element method and must be computed for the different types of elements.

Other approaches are based on wave propagation in periodic media. Wave propagation in waveguides and periodic structures was considered by many authors. Wave propagation in general periodic structures were studied by (Brillouin, 1953; Mead, 1973; Mead, 1975a; Mead, 1975b; Mead, 1996; Mead, 2009) using Floquet's theorem or transfer matrices. The waveguide finite element method (WFE) is similar to the SFE but the classical mass and stiffness matrices obtained by commercial finite element software can be used. So very complex waveguides can be modelled using the results of a finite element computation on only one period of the structure. This method is described in (Mace et al., 2005; Houillon et al., 2005; Duhamel et al., 2006; Duhamel, 2007) and consists in the analysis of wave propagation in periodic structures. This approach was applied to tyre vibration computations by (Nilsson, 2004; Waki et al., 2009). Classical methods for repetitive structures, for instance using cyclic periodicity, were also proposed by (Wang et al., 2003). In (Duhamel, 2009) a recursive method was proposed for the computation of frequency response functions of periodic structures. This method does not require the computation of wave functions and is computationally efficient.

In this paper the recursive method of (Duhamel, 2009) is applied to the computation of tyre vibrations for medium and high frequencies. In the first part the recursive method for curve periodic structures is described and its application to the computation of frequency response functions is explained. Then, examples of computations of tyre frequency response functions are presented before the conclusions.

\section{Vibration of periodic structures by the recursive method}

\subsection{Cell dynamics}

The periodic structure is divided into different cells as shown in Figure 1. A cell is described by a finite element model with an equal number of nodes on the left and right parts of the boundary. The discrete dynamic equation obtained by the finite element method for describing the movement of a cell is given, at the circular frequency $\omega$, by

$$
\left(\mathbf{K}+i \omega \mathbf{C}-\omega^{2} \mathbf{M}\right) \mathbf{q}=\mathbf{f}
$$

where $\mathbf{K}, \mathbf{M}$ and $\mathbf{C}$ are respectively the stiffness, mass and damping matrices, $\mathbf{f}$ is the loading vector and $\mathbf{q}$ the vector of the degrees of freedom (dofs). Introducing the dynamic stiffness matrix $\widetilde{\mathbf{D}}=\mathbf{K}+i \omega \mathbf{C}-\omega^{2} \mathbf{M}$, decomposing the dofs into the left 


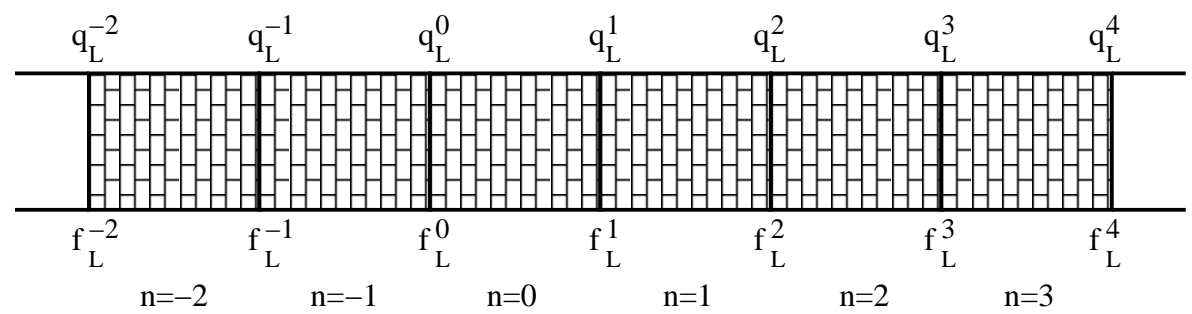

Figure 1. Periodic structure with the force and displacement vectors on the left and right parts of the boundary

$(L)$, right $(R)$ and interior $(I)$ sets, assuming there is no force on the interior dofs, results in

$$
\left[\begin{array}{ccc}
\widetilde{\mathbf{D}}_{I I} & \widetilde{\mathbf{D}}_{I L} & \widetilde{\mathbf{D}}_{I R} \\
\widetilde{\mathbf{D}}_{L I} & \widetilde{\mathbf{D}}_{L L} & \widetilde{\mathbf{D}}_{L R} \\
\widetilde{\mathbf{D}}_{R I} & \widetilde{\mathbf{D}}_{R L} & \widetilde{\mathbf{D}}_{R R}
\end{array}\right]\left[\begin{array}{c}
\mathbf{q}_{I} \\
\mathbf{q}_{L} \\
\mathbf{q}_{R}
\end{array}\right]=\left[\begin{array}{c}
\mathbf{0} \\
\mathbf{f}_{\mathbf{L}} \\
\mathbf{f}_{\mathbf{R}}
\end{array}\right]
$$

The interior dofs can be eliminated by using the first row of Equation [2] to get

$$
\mathbf{q}_{I}=-\widetilde{\mathbf{D}}_{I I}^{-1}\left(\widetilde{\mathbf{D}}_{I L} \mathbf{q}_{L}+\widetilde{\mathbf{D}}_{I R} \mathbf{q}_{R}\right)
$$

This leads to

$$
\left[\begin{array}{cc}
\widetilde{\mathbf{D}}_{L L}-\widetilde{\mathbf{D}}_{L I} \widetilde{\mathbf{D}}_{I I}^{-1} \widetilde{\mathbf{D}}_{I L} & \widetilde{\mathbf{D}}_{L R}-\widetilde{\mathbf{D}}_{L I} \widetilde{\mathbf{D}}_{I I}^{-1} \widetilde{\mathbf{D}}_{I R} \\
\widetilde{\mathbf{D}}_{R L}-\widetilde{\mathbf{D}}_{R I} \widetilde{\mathbf{D}}_{I I}^{-1} \widetilde{\mathbf{D}}_{I L} & \widetilde{\mathbf{D}}_{R R}-\widetilde{\mathbf{D}}_{R I} \widetilde{\mathbf{D}}_{I I}^{-1} \widetilde{\mathbf{D}}_{I R}
\end{array}\right]\left[\begin{array}{c}
\mathbf{q}_{L} \\
\mathbf{q}_{R}
\end{array}\right]=\left[\begin{array}{c}
\mathbf{f}_{L} \\
\mathbf{f}_{R}
\end{array}\right]
$$

or under a more compact form

$$
\left[\begin{array}{ll}
\mathbf{D}_{L L} & \mathbf{D}_{L R} \\
\mathbf{D}_{R L} & \mathbf{D}_{R R}
\end{array}\right]\left[\begin{array}{l}
\mathbf{q}_{L} \\
\mathbf{q}_{R}
\end{array}\right]=\left[\begin{array}{l}
\mathbf{f}_{L} \\
\mathbf{f}_{R}
\end{array}\right]
$$

This new dynamic stiffness matrix is obtained after the elimination of interior dofs. By the symmetry of the stiffness, mass and damping matrices the dynamic stiffness matrix of Equation [5] is also symmetric and we get ${ }^{t} \mathbf{D}_{L L}=\mathbf{D}_{L L},{ }^{t} \mathbf{D}_{R R}=\mathbf{D}_{R R}$ and ${ }^{t} \mathbf{D}_{L R}=\mathbf{D}_{R L}$ where ${ }^{t}$ is the transpose operator.

\subsection{Periodicity along a circle}

In this case, the dynamic stiffness matrix is not periodic in a Cartesian coordinate system. A possibility for getting a periodic system is to compute the matrices in a 
local cylindrical coordinate system $\left(\mathbf{e}_{r}, \mathbf{e}_{y}, \mathbf{e}_{\theta}\right)$ obtained by a rotation of the Cartesian system $\left(\mathbf{e}_{x}, \mathbf{e}_{y}, \mathbf{e}_{z}\right)$ by an angle $\theta$ around $\mathbf{e}_{y}$. The dynamic stiffness matrices of each portion of the periodic system will be identical in these new coordinates.

When a node is located at an angle $\theta$, one only needs to multiply the displacements and forces by a rotation matrix to get the new matrix in the coordinate system defined by $\left(\mathbf{e}_{r}, \mathbf{e}_{y}, \mathbf{e}_{\theta}\right)$. This rotation matrix is given by (for a rotation around the $y$ axis)

$$
\mathbf{R}(\theta)=\left[\begin{array}{ccc}
\cos \theta & 0 & \sin \theta \\
0 & 1 & 0 \\
-\sin \theta & 0 & \cos \theta
\end{array}\right]
$$

For each point $\mathbf{x}_{i}$, one needs the angle $\theta_{i}$ corresponding to this point to get the rotation. The new displacements are obtained, for instance, by

$$
\mathbf{q}_{i}^{P}=\mathbf{R}\left(\theta_{i}\right) \mathbf{q}_{i}^{C}
$$

where $P$ means the rotated system and $C$ the cartesian one. The discrete equation in the cartesian reference system is, with $\mathbf{D}_{C}$ denoting the dynamic stiffness matrix in this reference system,

$$
\mathbf{F}_{C}=\mathbf{D}_{C} \cdot \mathbf{Q}_{C}
$$

or, showing the contribution of each node,

$$
\left[\begin{array}{c}
\mathbf{R}_{1}^{-1}\left(\theta_{1}\right) \mathbf{f}_{1}^{P} \\
\mathbf{R}_{2}^{-1}\left(\theta_{2}\right) \mathbf{f}_{2}^{P} \\
\vdots \\
\mathbf{R}_{n}^{-1}\left(\theta_{n}\right) \mathbf{f}_{n}^{P}
\end{array}\right]=\mathbf{D}_{C}\left[\begin{array}{c}
\mathbf{R}_{1}^{-1}\left(\theta_{1}\right) \mathbf{q}_{1}^{P} \\
\mathbf{R}_{2}^{-1}\left(\theta_{2}\right) \mathbf{q}_{2}^{P} \\
\vdots \\
\mathbf{R}_{n}^{-1}\left(\theta_{n}\right) \mathbf{q}_{n}^{P}
\end{array}\right]
$$

which can also be written as

$$
\begin{aligned}
{\left[\begin{array}{l}
\mathbf{f}_{1}^{P} \\
\mathbf{f}_{2}^{P} \\
\vdots \\
\mathbf{f}_{n}^{P}
\end{array}\right]=} & {\left[\begin{array}{cccc}
\mathbf{R}_{1}\left(\theta_{1}\right) & \mathbf{0} & \cdots & \mathbf{0} \\
\mathbf{0} & \mathbf{R}_{2}\left(\theta_{2}\right) & \cdots & \mathbf{0} \\
\vdots & \vdots & \ddots & \vdots \\
\mathbf{0} & \mathbf{0} & \cdots & \mathbf{R}_{n}\left(\theta_{n}\right)
\end{array}\right] } \\
\times & \mathbf{D}_{C}\left[\begin{array}{cccc}
\mathbf{R}_{1}^{-1}\left(\theta_{1}\right) & \mathbf{0} & \cdots & \mathbf{0} \\
\mathbf{0} & \mathbf{R}_{2}^{-1}\left(\theta_{2}\right) & \cdots & \mathbf{0} \\
\vdots & \vdots & \ddots & \vdots \\
\mathbf{0} & \mathbf{0} & \cdots & \mathbf{R}_{n}^{-1}\left(\theta_{n}\right)
\end{array}\right]\left[\begin{array}{l}
\mathbf{q}_{1}^{P} \\
\mathbf{q}_{2}^{P} \\
\vdots \\
\mathbf{q}_{n}^{P}
\end{array}\right]
\end{aligned}
$$

In the new coordinates the transformed system is $\mathbf{F}_{P}=\mathbf{D}_{P} \cdot \mathbf{Q}_{P}$. Hence, one gets the relationship $\mathbf{D}_{P}=\mathbf{T} \cdot \mathbf{D}_{C} \cdot \mathbf{T}^{-\mathbf{1}}$ with

$$
\mathbf{T}=\left[\begin{array}{cccc}
\mathbf{R}_{1}\left(\theta_{1}\right) & \mathbf{0} & \cdots & \mathbf{0} \\
\mathbf{0} & \mathbf{R}_{2}\left(\theta_{2}\right) & \cdots & \mathbf{0} \\
\vdots & \vdots & \ddots & \vdots \\
\mathbf{0} & \mathbf{0} & \cdots & \mathbf{R}_{n}\left(\theta_{n}\right)
\end{array}\right]
$$


The other matrices have the same form and are given by

$$
\begin{aligned}
\mathbf{M}_{P} & =\mathbf{T} \cdot \mathbf{M}_{C} \cdot \mathbf{T}^{-1} \\
\mathbf{C}_{P} & =\mathbf{T} \cdot \mathbf{C}_{C} \cdot \mathbf{T}^{-\mathbf{1}}
\end{aligned}
$$

They have the same properties of symmetry as the original matrices. The relations linking the loading and displacement vectors in the two systems are

$$
\begin{aligned}
\mathbf{F}_{P} & =\mathbf{T} \cdot \mathbf{F}_{C} \\
\mathbf{Q}_{P} & =\mathbf{T} \cdot \mathbf{Q}_{C}
\end{aligned}
$$

\subsection{The recursive algorithm}

This algorithm consists in eliminating recursively the interior dofs. Consider a structure with a large number $N$ of cells. This number can be represented as a sum of powers of 2 as

$$
N=\sum_{i} 2^{p_{i}} \quad \text { with } \quad p_{1}>p_{2}>\cdots \geq 0
$$

where $p_{i}$ is the position of the $i_{t h}$ figure 1 in the binary representation of $N$, for instance $N=[1010 \ldots 01]_{2}$. Here, we want to compute the dynamic stiffness matrix for a structure with $N$ cells.

The dynamic stiffness matrices of two neighbouring cells are

$$
\mathbf{D}^{(\mathbf{1})}=\left[\begin{array}{cc}
\mathbf{D}_{L L}^{(1)} & \mathbf{D}_{L R}^{(1)} \\
\mathbf{D}_{R L}^{(1)} & \mathbf{D}_{R R}^{(1)}
\end{array}\right] ; \quad \mathbf{D}^{(\mathbf{2})}=\left[\begin{array}{cc}
\mathbf{D}_{L L}^{(2)} & \mathbf{D}_{L R}^{(2)} \\
\mathbf{D}_{R L}^{(2)} & \mathbf{D}_{R R}^{(2)}
\end{array}\right]
$$

Assembling the two matrices to get the dynamic stiffness matrix of the two-cells structure and eliminating the interior dofs leads to the equivalent matrix for the two-cells structure linking only the two boundaries.

$$
\begin{aligned}
\mathbf{D}^{\mathbf{e q}} & =\left[\begin{array}{cc}
\mathbf{D}_{L L}^{e q} & \mathbf{D}_{L R}^{e q} \\
\mathbf{D}_{R L}^{e q} & \mathbf{D}_{R R}^{e q}
\end{array}\right] \\
& =\left[\begin{array}{cc}
\mathbf{D}_{L L}^{(1)}-\mathbf{D}_{L R}^{(1)} \mathbf{D}^{*} \mathbf{D}_{R L}^{(1)} & -\mathbf{D}_{L R}^{(1)} \mathbf{D}^{*} \mathbf{D}_{L R}^{(2)} \\
-\mathbf{D}_{L R}^{(2)} \mathbf{D}^{*} \mathbf{D}_{R L}^{(1)} & \mathbf{D}_{R R}^{(2)}-\mathbf{D}_{R L}^{(2)} \mathbf{D}^{*} \mathbf{D}_{L R}^{(2)}
\end{array}\right]
\end{aligned}
$$

with $\mathbf{D}^{*}=\left[\mathbf{D}_{R R}^{(1)}+\mathbf{D}_{L L}^{(2)}\right]^{-1}$.

Continuing this process, one can compute the equivalent matrices for structures with $2,4, \ldots, 2^{n_{b}-1}$ cells where $n_{b}$ is the length of the binary representation of $N$. Assembling the matrices corresponding to the $p_{i}$ figures and eliminating the interior 


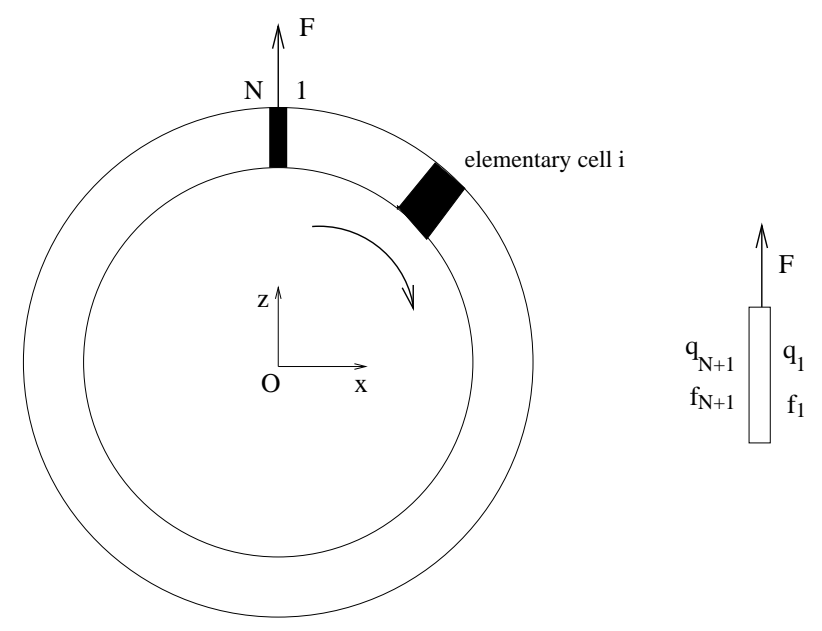

Figure 2. Model of the tyre

dofs leads to the equivalent matrix for the global structure. The advantage of this method is that the computing time is proportional to $\log N$, see (Duhamel, 2009) for more details.

\subsection{Computation of the frequency response function}

After the elimination of all the interior dofs, the discrete relation for the whole structure between sections 1 and $N+1$ is

$$
\left[\begin{array}{c}
\mathbf{f}_{1} \\
\mathbf{f}_{N+1}
\end{array}\right]=\left[\begin{array}{ll}
\mathbf{D}_{11}^{T} & \mathbf{D}_{12}^{T} \\
\mathbf{D}_{21}^{T} & \mathbf{D}_{22}^{T}
\end{array}\right]\left[\begin{array}{c}
\mathbf{q}_{1} \\
\mathbf{q}_{N+1}
\end{array}\right]
$$

The conditions of continuity of the displacements and the equilibrium of forces on the section where the load is applied lead to (see Figure 2)

$$
\left\{\begin{array}{l}
\mathbf{q}_{1}=\mathbf{q}_{N+1}=\mathbf{q} \\
\mathbf{f}_{1}+\mathbf{f}_{N+1}+\mathbf{F}=\mathbf{0}
\end{array}\right.
$$

From Relations [19] and [20], one gets

$$
\begin{aligned}
-\mathbf{F} & =\mathbf{f}_{1}+\mathbf{f}_{N+1} \\
& =\mathbf{D}_{11}^{T} \mathbf{q}_{1}+\mathbf{D}_{12}^{T} \mathbf{q}_{N+1}+\mathbf{D}_{21}^{T} \mathbf{q}_{1}+\mathbf{D}_{22}^{T} \mathbf{q}_{N+1} \\
& =\left(\mathbf{D}_{11}^{T}+\mathbf{D}_{12}^{T}+\mathbf{D}_{21}^{T}+\mathbf{D}_{22}^{T}\right) \mathbf{q}
\end{aligned}
$$

Denoting $\mathbf{D}_{11}^{T}+\mathbf{D}_{12}^{T}+\mathbf{D}_{21}^{T}+\mathbf{D}_{22}^{T}=\mathbf{D}_{\text {tot }}$, one gets the dynamic equation

$$
\mathbf{D}_{\text {tot }} \mathbf{q}=-\mathbf{F}
$$


Table 1. Mechanical properties of the materials of the tyre

\begin{tabular}{|c|c|c|c|c|}
\hline & $\rho$ & $E$ & $\nu$ & $\eta$ \\
\hline Rubber & $1000 \mathrm{~kg} / \mathrm{m}^{3}$ & $2810^{6} \mathrm{~Pa}$ & 0.48 & 0.1 \\
\hline Steel & $7850 \mathrm{~kg} / \mathrm{m}^{3}$ & $210^{11} \mathrm{~Pa}$ & 0.33 & 0 \\
\hline Tread & $2500 \mathrm{~kg} / \mathrm{m}^{3}$ & $40010^{6} \mathrm{~Pa}$ & 0.4 & 0.1 \\
\hline
\end{tabular}

The solution of this equation gives the displacements on the section. By postprocessing, the solution in the whole structure can be obtained by assembling and solving relations equivalent to Equation [22] for various parts of the structure.

\section{Application to tyre vibrations}

\subsection{Model of a tyre}

A tyre is considered as a structure with a rotational symmetry. A section of a tyre is composed of different materials, for instance the belt is made of steel wires with rubber. Moreover the geometry is complex and only a finite element model can describe correctly the details of this structure. The geometrical parameters of a typical tyre are presented in Figure 3 where $R=0.278 \mathrm{~m}$ is the exterior radius and $r=0.168 \mathrm{~m}$ the interior radius. The geometry of this tyre is identical to that of the Michelin "energy 165/65R13". The section, the mesh and the material distribution are also presented in Figure 3 for a periodic cell. The material properties are given in Table 1 where $E, \nu, \rho, \eta$ are respectively the Young's modulus, the Poisson coefficient, the density and the loss factor. Some simplifications have been made on the material properties because the real purpose here is to illustrate the possibilities of the method and not to consider with great details the whole complexity of the tyre model.

Two cases are considered, without and with pres-stress. In the first case, the internal air-pressure is neglected. In the second case, the influence of the internal airpressure on the stiffness of the structure is considered. This pressure creates an initial static stress field $\sigma^{0}$, an initial displacement $\mathbf{q}^{0}$ and the dynamic movement is supposed to be a small perturbation around this static solution. The displacements, deformations and stresses can be decomposed as

$$
\begin{aligned}
& \mathbf{q}=\mathbf{q}^{0}+\mathbf{q}^{*} \\
& \varepsilon=\varepsilon^{0}+\varepsilon^{*} \\
& \sigma=\sigma^{0}+\sigma^{*}
\end{aligned}
$$

where ${ }^{0}$ means the static solution, ${ }^{*}$ is the dynamic perturbation and the left hand sides of the relationship give the total mechanical fields. Globally, this leads to a new model with a modified dynamic stiffness matrix given by $\mathbf{D}=\left[\mathbf{K}+\mathbf{K}_{G}\right]+i \omega \mathbf{C}-\omega^{2} \mathbf{M}$ with the new stiffness matrix $\left[\mathbf{K}+\mathbf{K}_{G}\right]$. The matrix $\mathbf{K}_{G}$, which is the contribution 


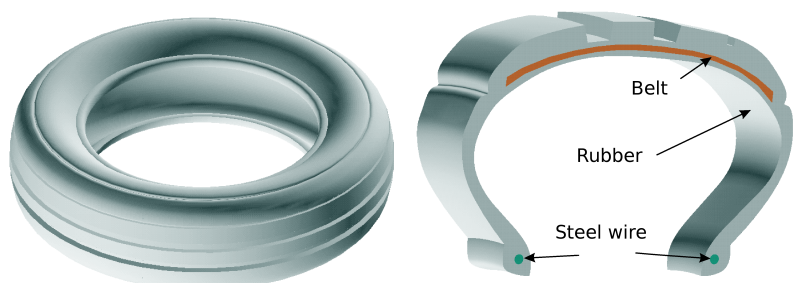

(a)

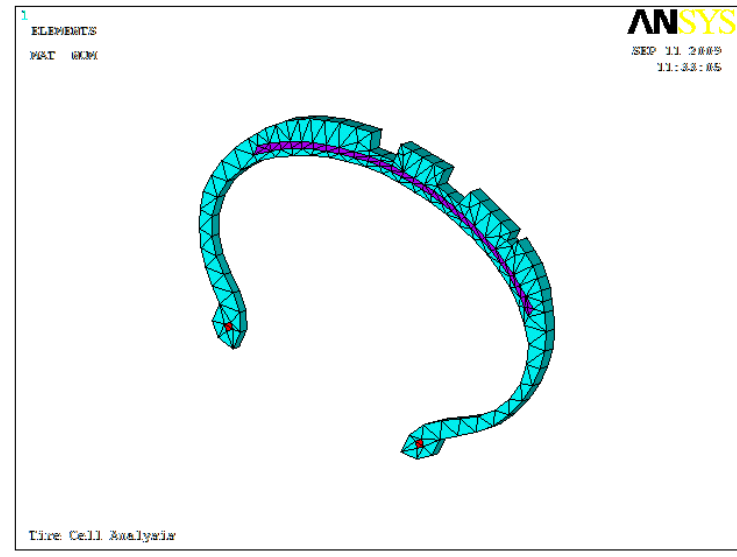

(b)

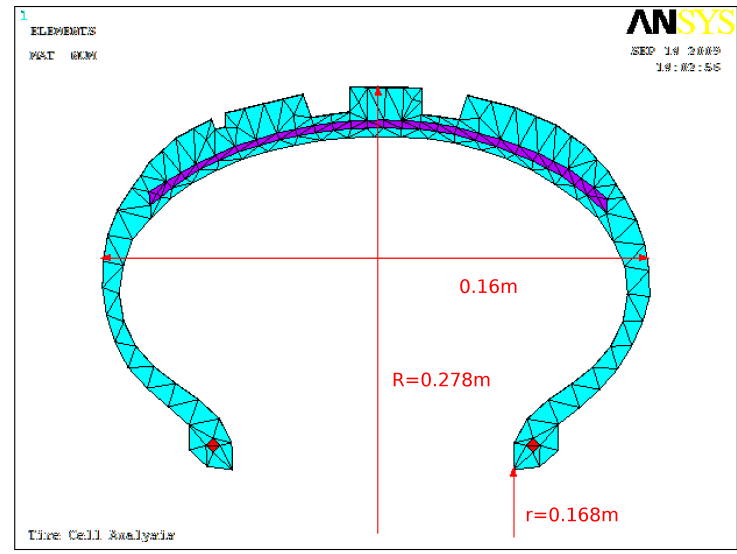

(c)

Figure 3. (a) Global geometry, (b) mesh of a period and (c) dimensions and materials in a section of the tyre. The materials are : rubber in blue, steel reinforced rubber in magenta and steel in red 
of the internal pressure to the stiffness, can also be obtained by Ansys. Appropriate boundary conditions must be applied at both end sections to get a solution with circular symmetry on a period for the static solution under the influence of the air-pressure. These sections should have planar displacements and the angle between these sections should be the same as in the initial mesh so that the displacement will be only radial as it would be if the complete tyre had been computed.

\subsection{Homogeneous section}

To test the method, we first consider the simple case of a tyre with a homogeneous section. The mesh is obtained by Ansys with an equal number of nodes on the two opposite sections of the period and the nodes at the rim are fixed in the modelling considered here. There is a total of 116 nodes, 58 on each section as there is no interior node in this example and globally this leads to 348 dofs. The mesh is made with 28 elements of type solid45 from Ansys with 8 nodes. The material is linear isotropic. Anisotropic elements would allow a better modelling of the tyre but, as this does not change the number of dofs, only isotropic elements are used here. The material properties here are $E=80 M P a$ for the Young modulus, $\nu=0.42$ for the Poisson coefficient, $\rho=1200 \mathrm{~kg} / \mathrm{m}^{3}$ for the density and $\eta=0.1$ for the damping coefficient. The nodes in contact with the rim are fixed. A point force is applied in the middle of the tread and the mobilities are computed for various points on the tyre surface. These points are shown in Figure 4 for a transverse section of the tyre. Figure 5 shows the comparison of the full three dimensional analysis with Ansys and the computation with the periodic model. The full three dimensional model is made of the same elements as the periodic section with a total of 2610 nodes and 7830 dofs. The curves represent the radial and transverse point mobilities at the position of the force, respectively. The transverse mobility is obtained with a force tangential to the tyre along the circumferential direction and with a response in the same direction. It can be observed that the two results are very close. The periodic model allows computations up to $2000 \mathrm{~Hz}$ without difficulties while the Ansys computations have been limited to low frequencies to be compatible with a reasonable computing time. The maximal relative error between the periodic and full three-dimensional solutions has been found to be of order $410^{-6}$ in the axial case. So no significant loss in precision is found by using the periodic method instead of the usual finite element solution.

In Figure 6, the influence of the angle of the periodic cell is presented. The force is located as in the precedent example but the response is computed at point 5 of Figure 4. As expected the influence of the angular aperture is important only for high frequencies. One can also observe that the influence is higher for the radial component than for the tangential part. The solution is not strongly dependent on the angle of the cell as long as this angle is small enough for the frequency of the computation. 


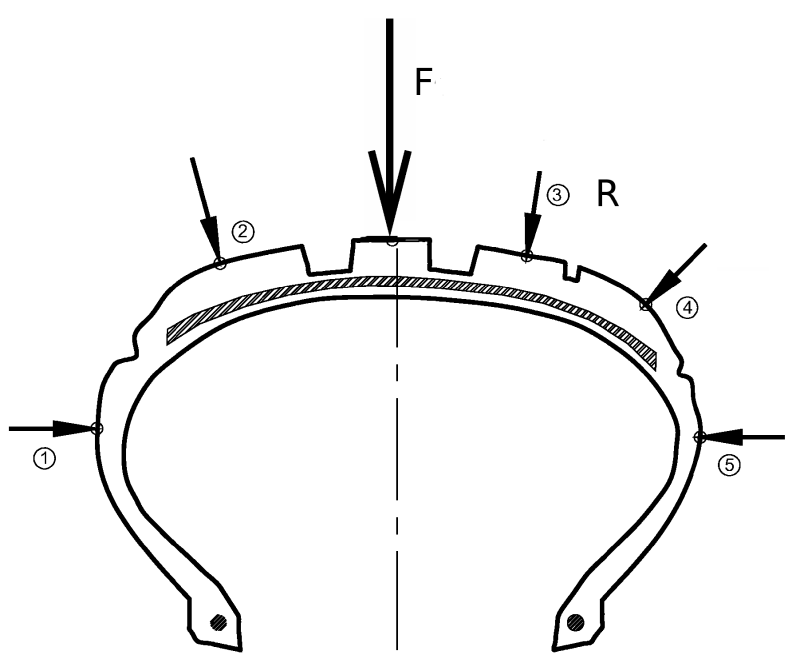

Figure 4. Position of the excitation and of the response points

\subsection{Tyre with a more complex section}

The periodic cell shown in Figure 3 is used to model the real tyre. It is made of three materials : rubber, steel reinforced rubber and steel with the properties given in Table 1. To simplify the computation, the steel reinforced rubber has been considered as isotropic, while a more detailed analysis should consider the anisotropy of this material. A first computation is made without internal pressure with the mesh shown in Figure 3. The angle of a period is $6^{\circ}$ and the nodes near the rim are fixed. In Figure 7 the computation of the mobility at the position of the force and at the points shown in Figure 4 are presented for the present method. The responses are close for low frequencies except at the position of the force. The differences increase with the frequency.

Another set of results is presented with an internal air-pressure of $210^{5} \mathrm{~Pa}$. The results are shown in Figure 8 for the periodic model and for the 3D modal synthesis over 1000 modes. There is a perfect agreement over the frequency range where the two curves can be compared. Results agree very well up to $800 \mathrm{~Hz}$ which is the limit of the 3D results, while the present method allows an efficient computation up to 2000 Hz.

The computing time, on a PC with a core 2 quad processor and 4GB of memory, can be estimated to an average of 10s per frequency with the periodic method while the 3D computation with Ansys needs 133 seconds per frequency by the direct method and about 3 s for the modal synthesis, see Table 2. However in this last case, it was not 
20 EJCM - 20/2011. Dynamics of materials, structures and systems

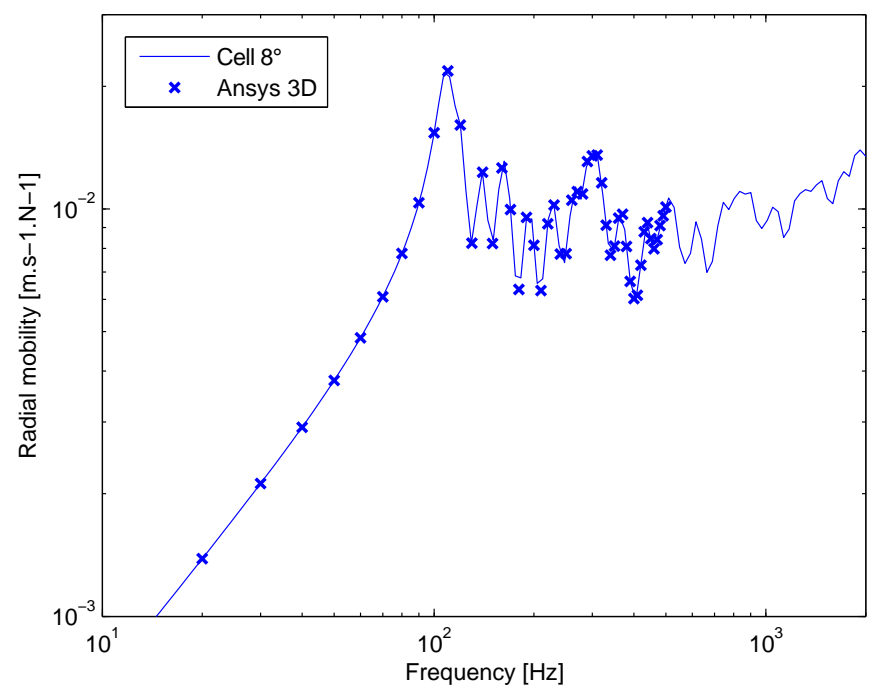

(a)

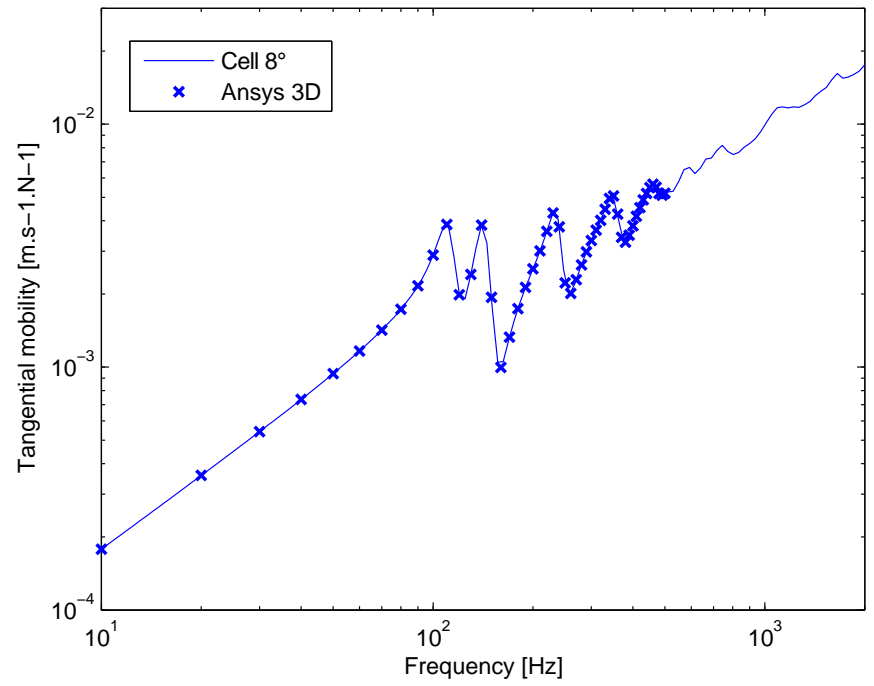

(b)

Figure 5. Comparison of the present technique with a full Ansys computation for the radial $(a)$ and tangential mobilities $(b)$ 


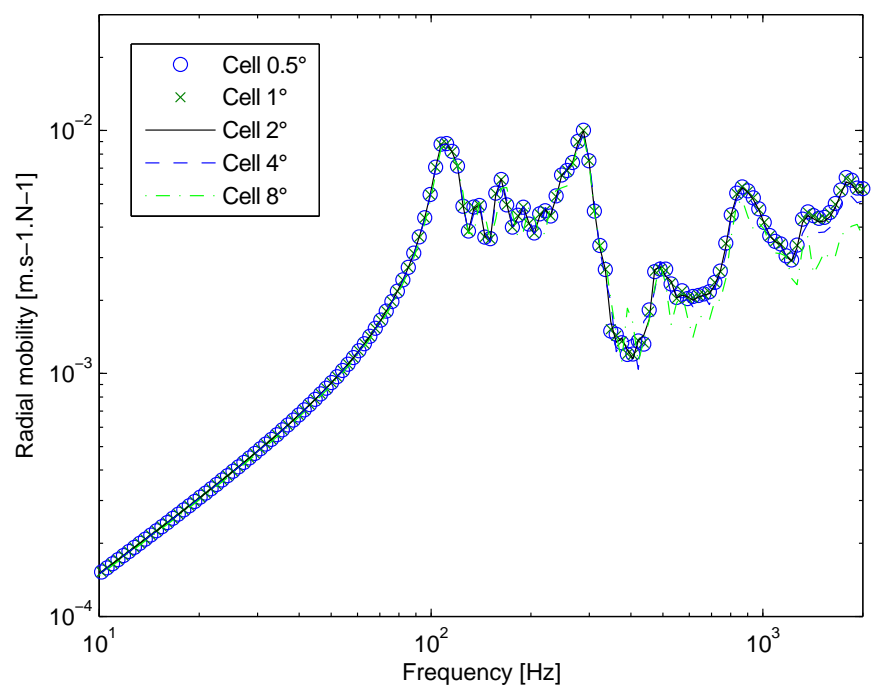

(a)

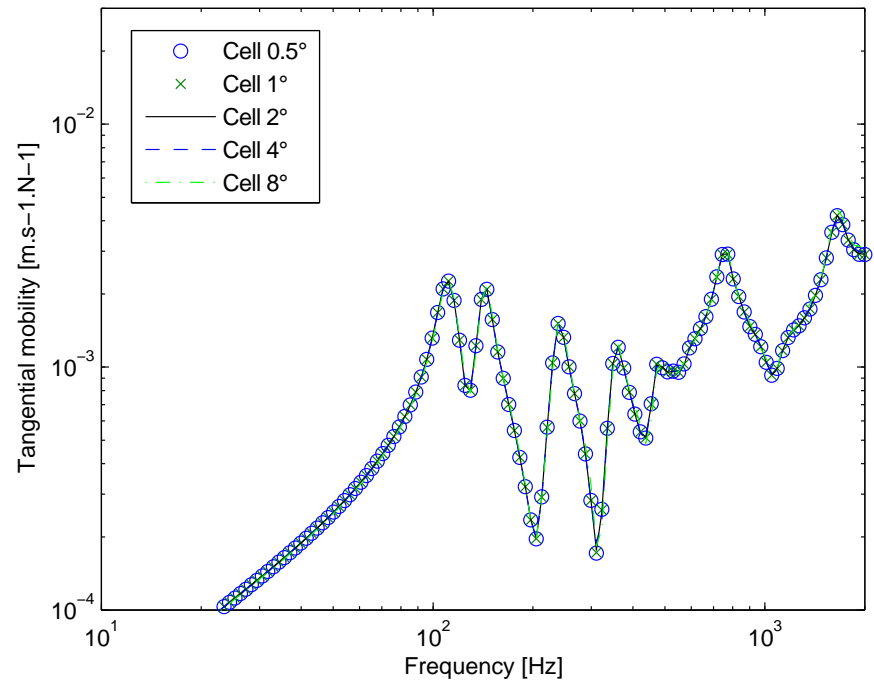

(b)

Figure 6. Comparison of the radial (a) and tangential (b) mobilities for periods with angles $0.5^{\circ}, 1^{\circ}, 2^{\circ}, 4^{\circ}, 8^{\circ}$ and an excitation at the middle of the belt 


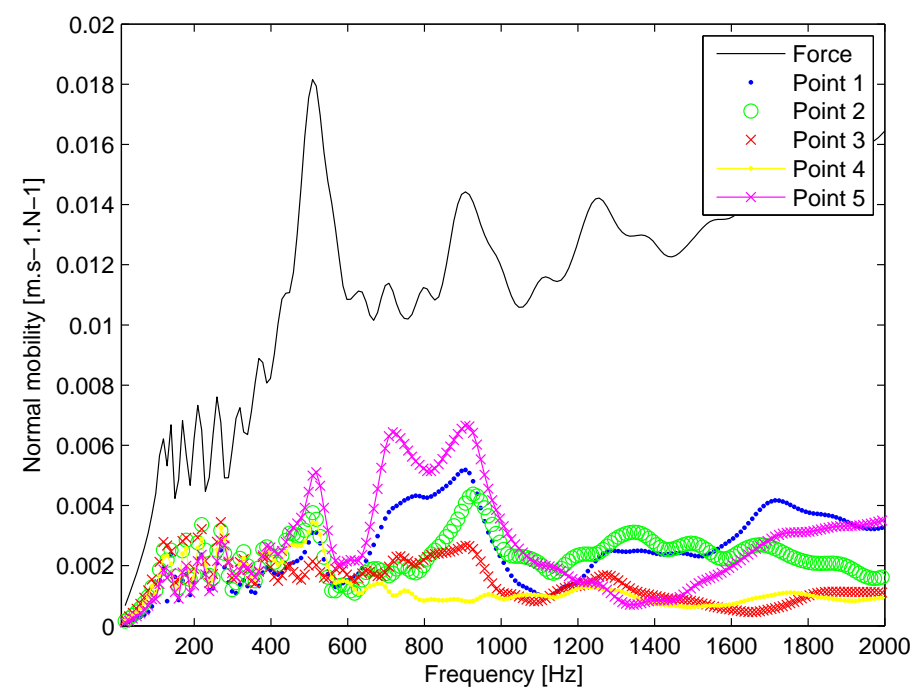

Figure 7. The FRF of the normal mobilities at different points in a section

Table 2. CPU times and data sizes

\begin{tabular}{|c|c|c|}
\hline Method & CPU Times per frequency & Data size \\
\hline Ansys 3D direct solver & $133 \mathrm{~s}$ & $26 \mathrm{~GB}$ \\
\hline Ansys 3D with 1000 modes & $3 \mathrm{~s}$ & $28 \mathrm{~GB}$ \\
\hline Recursive method & $10 \mathrm{~s}$ & $2 \mathrm{MB}$ \\
\hline
\end{tabular}

possible to compute with more than 1000 modes because the requirements in memory and disk size were larger than the possibility of the computer. So, in practise, the results and comparisons could only be provided for low frequencies for which the modal synthesis is faster but this method cannot give results for frequencies larger than $1000 \mathrm{~Hz}$ or for meshes with a higher density than whose used in the examples presented here. Concerning the volume of data with a mesh of the total tyre with 60 periodic cells, each with 173 nodes, the Ansys result file size is $28 \mathrm{~GB}$ by 3D methods while only $2 \mathrm{MB}$ are needed by the present method because the size is independent of the number of cells. 


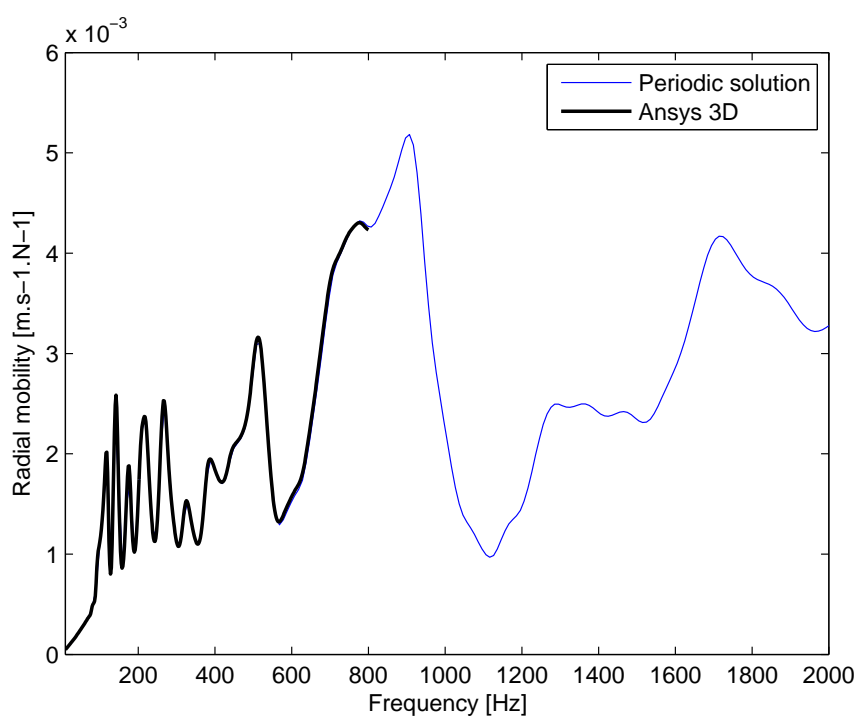

(a)

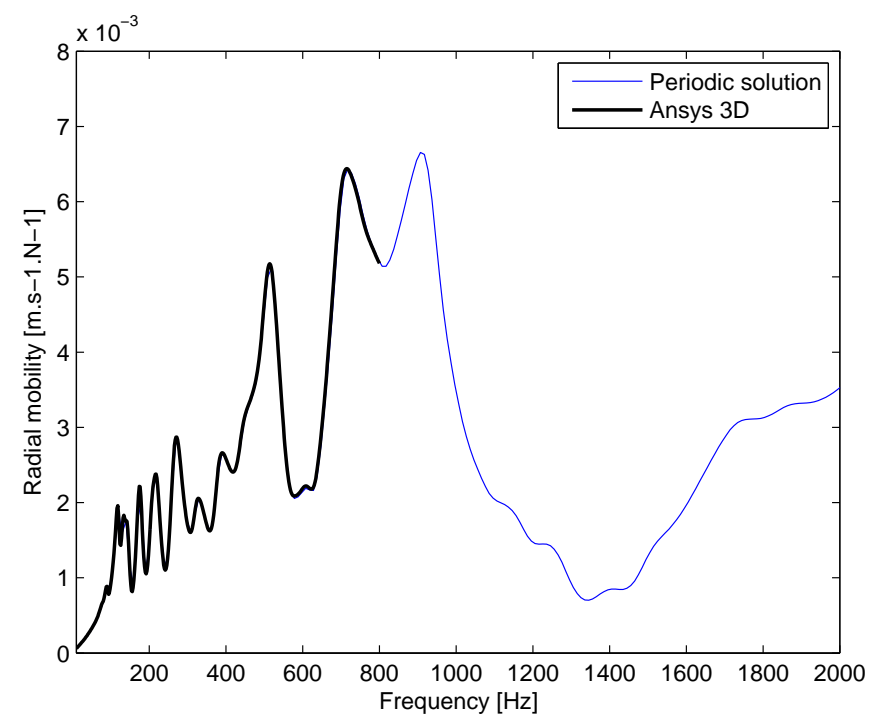

(b)

Figure 8. Comparison of the FRF with the Ansys modal synthesis for the tyre with an internal pressure at point 1 in direction $y(a)$ and at point 5 in direction $y(b)$ 
24 EJCM - 20/2011. Dynamics of materials, structures and systems

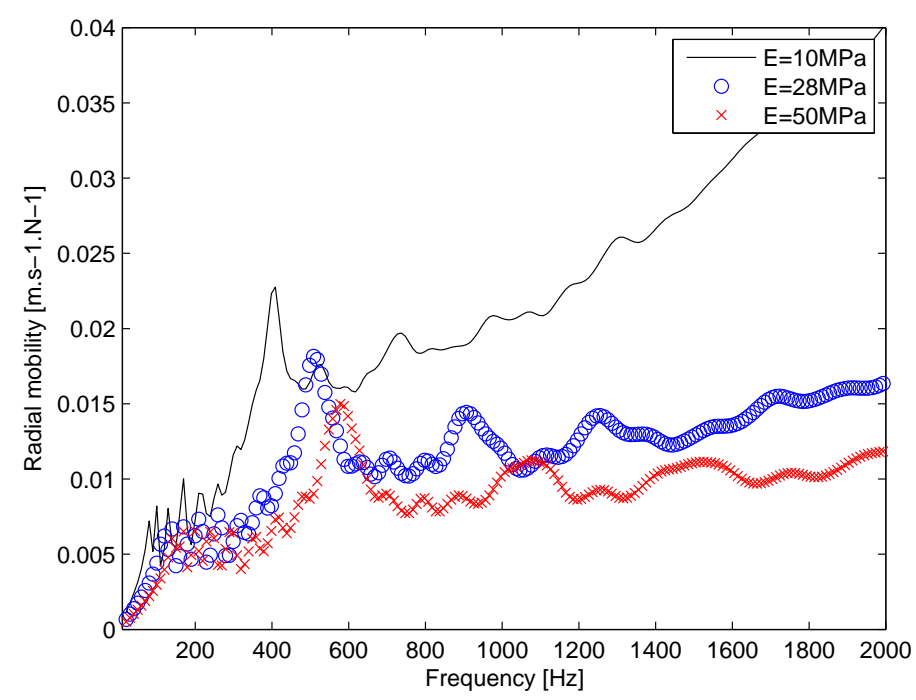

Figure 9. Influence of the modulus of the rubber on the radial mobility at the position of the force

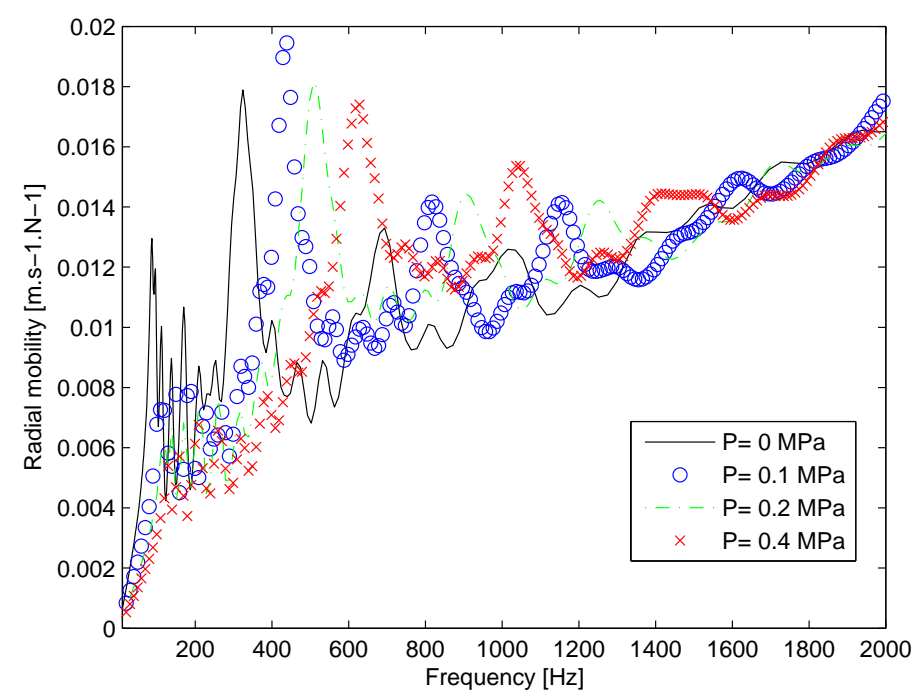

Figure 10. Influence of the internal air-pressure on the mobility 


\subsection{Influence of some parameters}

The tyre has a large volume of rubber. Its properties should have a great influence on the dynamic response of the tyre. In Figure 9 the mobilities are presented for three different values of the modulus of the rubber. As expected, the mobilities decreases with the value of the rubber modulus.

In Figure 10 this influence is presented on the mobility for the frequency range $[0-3000 \mathrm{~Hz}]$. It can be observed that changing the pressure mainly results in a shift in the resonance frequencies which comes from the increase in rigidity as the internal pressure increases.

\section{Conclusions}

The computation of vibrations of a real tyre with the details of the material properties and of the geometry is presented using a recursive method based on a finite element modelling. Only a small part of the tyre needs to be meshed and the computation by the recursive algorithm allows the determination of mobilities at different points on the tyre over a much larger frequency range than the traditional three dimensional analysis. A parametric study is possible showing, for instance, the influence of the rubber Young modulus or of the internal air-pressure on the response of the tyre at different points.

\section{References}

Bartoli I., Marzani A., di Scalea F., Viola E., Modeling wave propagation in damped waveguides of arbitrary cross-section , Journal of Sound and Vibration, vol. 295, p. 685-707, 2006.

Brillouin L., Wave propagation in periodic structures, New York, Dover, 1953.

Brinkmeier M., Nackenhorst U., Petersen S., vonEstorff O., A finite element approach for the simulation of tire rolling noise., Journal of Sound and Vibration, vol. 309, p. 20-39, 2008.

Campanac P., Nonami K., Duhamel D., Application of the vibration analysis of linear systems with time-periodic coefficients to the dynamics of a rolling tyre, Journal of Sound and Vibration, vol. 231, n 1 , p. 37-77, 2000.

Duhamel D., Finite element computation of Green's functions, Engineering Analysis with Boundary Elements, vol. 31, n 11, p. 919-930, 2007.

Duhamel D., A recursive approach for the finite element computation of waveguides, Journal of Sound and Vibration, vol. 323, $\mathrm{n}^{\circ}$ 1-2, p. 163-172, 2009.

Duhamel D., Mace B., Brennan M., Finite element analysis of the vibrations of waveguides and periodic structures., Journal of Sound and Vibration, vol. 294, p. 205-220, 2006.

Finnveden S., Formulas for modal density and for input power from mechanical and fluid point sources in fluid filled pipes, Journal of Sound and Vibration, vol. 208, $\mathrm{n}^{\circ}$ 5, p. 705-728, 1997a. 
Finnveden S., Spectral finite element analysis of the vibration of straight fluid-filled pipes with flanges, Journal of Sound and Vibration, vol. 199, p. 125-154, 1997b.

Hamet J. F., Tire/road noise: time domain Green's function for the orthotropic plate model , Acta acoustica united with Acustica, vol. 87, p. 470-474, 2001.

Heckl M., Tyre noise generation, Wear, vol. 113, p. 157-170, 1986.

Houillon L., Ichchouh M. N., Jezequel L., Wave motion in thin-walled structures , Journal of Sound and Vibration, vol. 281, p. 483-507, 2005.

Huang S., Soedel W., Response of rotating rings to harmonic and periodic loading and comparison with the inverted problem, Journal of Sound and Vibration, vol. 118, $n^{\circ} 2$, p. 253-270, 1987.

Kim Y., Bolton J., Effects of rotation on the dynamics of a circular cylindrical shell with application to tire vibration, Journal of Sound and Vibration, vol. 275, p. 605-621, 2004.

Kozhevnikov I., The vibrations of a free and loaded tyre , Journal of Applied Mathematics and Mechanics, vol. 70, p. 223-228, 2006.

Kropp W., Structure-borne sound on a smooth tyre , Applied Acoustics., vol. 26, p. 181-192, 1989.

Larsson K., Barrelet S., Kropp W., The modelling of the dynamic behavior of tyre tread blocks , Applied Acoustics, vol. 63, p. 659-677, 2002a.

Larsson K., Kropp W., A high-frequency three-dimensional tyre model based on two coupled elastic layers, Journal of Sound and Vibration, vol. 253, n 4, p. 889-908, 2002 b.

Lopez I., Blom R., Roozen N., Nijmeijer H., Modelling vibrations on deformed rolling tyres a modal approach , Journal of Sound and Vibration, vol. 307, p. 481-494, 2007.

Mace B., Duhamel D., Brennan M., Hinke L., Finite element prediction of wave motion in structural waveguides, The Journal of the Acoustical Society of America, vol. 117, $\mathrm{n}^{\circ} 5$, p. 2835-2843, 2005.

Marzani A., Viola E., Bartoli I., di Scalea F., Rizoo P., A semi-analytical finite element formulation for modeling stress wave propagation in axisymmetric damped waveguides , Journal of Sound and Vibration, vol. 318, p. 488-505, 2008.

Mead D., A general theory of harmonic wave propagation in linear periodic systems with multiple coupling., Journal of Sound and Vibration, vol. 27, p. 235-260, 1973.

Mead D., Wave propagation and natural modes in periodic systems: I. Mono-coupled systems, Journal of Sound and Vibration, vol. 40, p. 1-18, 1975a.

Mead D., Wave propagation in continuous periodic structures: research contributions from Southampton, 1964-1995 , Journal of Sound and Vibration, vol. 190, p. 495-524, 1996.

Mead D., The forced vibration of one-dimensional multi-coupled periodic structures: An application to finite element analysis. , Journal of Sound and Vibration, vol. 319, p. 282-304, 2009.

Mead D. J., Wave propagation and natural modes in periodic systems: II. Multi-coupled systems, with and without damping., Journal of Sound and Vibration, vol. 40, p. 19-39, 1975 b.

Molisani L., Burdisso R., Tsihlas D., A coupled tire structure/acoustic cavity model , International Journal of Solids and Structures, vol. 40, p. 5125-5138, 2003.

Muggleton J., Mace B., Brennan M., Vibrational response prediction of a pneumatic tyre using an orthotropic two-plate wave model , Journal of Sound and Vibration, vol. 264, p. 929-950, 2003. 
Nilsson C., Waveguide finite elements applied on a car tyre, PhD thesis, KTH Royal Institute of Technology, 2004.

Pinnington R., Radial force transmission to the hub from an unloaded stationary tyre , Journal of Sound and Vibration, vol. 253, $\mathrm{n}^{\circ}$ 5, p. 961-983, 2002.

Pinnington R., A wave model of a circular tyre. Part 1: belt modelling , Journal of Sound and Vibration, vol. 290, p. 101-132, 2006 .

Pinnington R., A wave model of a circular tyre. Part 2: side-wall and force transmission modelling , Journal of Sound and Vibration, vol. 290, p. 133-168, $2006 \mathrm{~b}$.

Pinnington R., Briscoe A., A wave model for a pneumatic tyre belt, Journal of Sound and Vibration, vol. 253, n 5 , p. 941-959, 2002.

Waki Y., Mace B., Brennan M., Free and forced vibrations of a tyre using a wave/finite element approach. , Journal of Sound and Vibration, vol. 323, p. 737-756, 2009.

Wang D., Zhou C., Rong J., Free and forced vibration of repetitive structures , International Journal of Solids and Structures, vol. 40, p. 5477-5494, 2003. 
